\title{
Mammary analog secretory carcinoma of the thyroid gland: A primary thyroid adenocarcinoma harboring ETV6-NTRK3 fusion
}

Snjezana Dogan ${ }^{1}$, Lu Wang ${ }^{1}$, Ryan N Ptashkin ${ }^{1}$, Robert R Dawson ${ }^{2}$, Jatin P Shah ${ }^{3}$, Eric J Sherman ${ }^{4}$, R Michael Tuttle ${ }^{4}$, James A Fagin ${ }^{4}$, David S Klimstra ${ }^{1}$, Nora Katabi ${ }^{1}$ and Ronald A Ghossein ${ }^{1}$

${ }^{1}$ Department of Pathology, Memorial Sloan Kettering Cancer Center, New York, NY, USA; ${ }^{2}$ Department of Pathology, Good Samaritan Hospital, West Islip, NY, USA; ${ }^{3}$ Department of Surgery, Memorial Sloan Kettering Cancer Center, New York, NY, USA and ${ }^{4}$ Department of Medicine, Memorial Sloan Kettering Cancer Center, New York, NY, USA

\begin{abstract}
ETV6-NTRK3 fusion was identified in several cancers including the recently described mammary analog secretory carcinoma (MASC) of the salivary glands and a minority of papillary thyroid carcinomas. We describe three cases of primary MASC of the thyroid gland and provide a detailed clinical and pathological characterization of the tumor morphology, immunoprofile, and genetic background. Immunohistochemistry for PAX8, TTF-1, thyroglobulin, mammaglobin, GCDFP-15, S-100 protein, and p63 was used to define the tumor immunophenotype. Fluorescence in situ hybridization for ETV6 rearrangement was performed in three, and the next-generation sequencing assay MSK-IMPACT ${ }^{\mathrm{TM}}$ (Memorial Sloan Kettering-Integrated Mutation Profiling of Actionable Cancer Targets) was performed in two cases. Primary MASC of the thyroid occurred in two women and one man, age 47-72 years. All patients presented with high T stage, infiltrative, locally aggressive tumors with extrathyroidal extension. Two cases were associated with well-differentiated papillary thyroid carcinoma. Histologically, they appeared as low-grade tumors, resembling MASC of the salivary glands and labeled positive for mammaglobin, GCDFP-15, S-100 protein, p63, weakly positive for PAX8, and negative for TTF-1 and thyroglobulin. Fluorescence in situ hybridization revealed ETV6 rearrangement in all cases. In two tested cases MSK-IMPACT $^{\mathrm{TM}}$ confirmed the presence of ETV6-NTRK3 gene fusion. Two patients had at least two local recurrences, one was alive with disease, and one was alive and free of disease after 14 and 17 years, respectively. The third patient was alive and free of disease after 2 years. MASC of the thyroid is histologically, immunophenotypically, and genetically similar to its salivary gland counterpart. Thyroid MASC can be associated with a well-differentiated papillary thyroid carcinoma component, supporting follicular cell origin. Clinically, these carcinomas may show frequent recurrences but are associated with long-term survival. Patients with MASC of the thyroid may potentially benefit from Trk molecular-targeted therapy.

Modern Pathology (2016) 29, 985-995; doi:10.1038/modpathol.2016.115; published online 10 June 2016
\end{abstract}

Mammary analog secretory carcinoma (MASC) of the salivary glands was first described by Skálová et al. ${ }^{1}$ as a carcinoma that markedly resembles secretory carcinoma of the breast at the histological and

Correspondence: Dr S Dogan, MD, Department of Pathology Memorial Sloan Kettering Cancer Center, 1275 York Avenue, New York, NY 10065, USA.

E-mail: dogans@mskcc.org

Received 30 March 2016; revised 19 April 2016; accepted 2 May 2016; published online 10 June 2016 immunohistochemical levels. Similar to its breast counterpart, MASC harbors a recurrent balanced chromosomal translocation $\mathrm{t}(12 ; 15)(\mathrm{p} 13 ; \mathrm{q} 25)$ leading to ETV6-NTRK3 gene fusion. ${ }^{1,2}$ ETV6-NTRK3 fusion was also detected in several other entities such as infantile fibrosarcoma, congenital mesoblastic nephroma, and acute myeloid leukemia. ${ }^{3-5}$ More recently, ETV6-NTRK3 fusion was found in about $14 \%$ of radiation-induced well-differentiated papillary thyroid carcinomas, and in up to $2 \%$ of sporadic well-differentiated papillary thyroid carcinomas. ${ }^{6,7}$ 
Aside from mucoepidermoid carcinoma and its variant sclerosing mucoepidermoid carcinoma with eosinophilia, no other primary salivary gland-type tumors of the thyroid have been reported. ${ }^{8-10}$ To the best of our knowledge, we describe here the first three cases of a salivary gland-type adenocarcinoma primary to the thyroid harboring ETV6-NTRK3 fusion and markedly resembling the morphology and immunoprofile of the MASC of the salivary glands.

\section{Case 1}

A 72-year-old woman, a former heavy smoker, without history of radiation exposure or breast cancer underwent total thyroidectomy for a right lobe mass measuring $2.9 \mathrm{~cm}$ in greatest dimension, invading the trachea, soft tissue and skeletal muscle of the neck. Microscopically the tumor was infiltrative and was interpreted as 'moderately differentiated papillary thyroid carcinoma'. The tumor was staged as a T4 No Mo (stage III), and the surgical margin was focally positive for tumor. Before her surgery she had an undetectable serum thyroglobulin level and elevated thyroid-stimulating hormone. However, given the high-risk features at presentation, she was subsequently treated with radioactive iodine (Table 1). Over the next 14 years the patient had two recurrences to the soft tissue and skin of the left neck, each time with low or undetectable serum thyroglobulin levels. The first recurrence was interpreted as metastatic papillary thyroid carcinomas, whereas in the second a possibility of a metastasis from 'a primary salivary gland-type adenocarcinoma originating in the thyroid, in salivary gland rests adjacent to thyroid, or minor salivary glands adjacent to trachea' was entertained based on extensive diagnostic work-up (Table 2). In the meantime, 1 year after the first recurrence, she was also found to have multiple bilateral lung nodules, which ultimately proved to represent primary lung adenocarcinomas based on immunolabeling for PE10 and the finding of a KRAS G12C mutation, which is typically associated with smoking-related lung adenocarcinoma. However, given the lack of aggressive biological features and the overall favorable performance status, no further treatment was indicated at that time and she was not treated for the lung carcinomas. At the last follow-up, the patient was alive without thyroid-related disease 17 years after initial presentation (Table 1).

\section{Case 2}

A 47-year-old man without history of radiation exposure underwent total thyroidectomy with a limited left neck lymph node dissection for a left lobe tumor. The $7.0 \mathrm{~cm}$ tumor was replacing the left lobe, showed extrathyroidal extension into perithyroidal fibroadipose tissue and was diagnosed as a 'well-differentiated papillary thyroid carcinoma with tall cell features'. Surgical margins were free of tumor and his left neck lymph nodes were positive for metastatic carcinoma with extranodal extension. Over a period of 14 years the thyroid carcinoma recurred locally three times, each time with undetectable serum thyroglobulin levels (Table 1). The first local recurrence was interpreted as metastatic papillary thyroid carcinomas, and when the tumor recurred second time an extensive diagnostic workup was performed suggesting that the primary thyroid tumor might have represented 'a salivary gland-type adenocarcinoma with MASC-like features, originating in the thyroid, in salivary gland rests adjacent to thyroid or minor salivary glands adjacent to trachea' (Table 2). Next time the patient presented with enlarged residual left neck lymph nodes which suggested third recurrence. However, given the potential surgical risk, observation was recommended until the molecular testing confirmed the presence of ETV6-NTRK3 fusion and he was considered for a clinical trial with Trk inhibitors (Table 1).

\section{Case 3}

A 65-year-old woman without history of radiation exposure or breast cancer underwent total thyroidectomy for an isthmic mass at an outside hospital. There was a $6.5 \mathrm{~cm}$ tumor in the isthmus with extrathyroidal extension into perithyroidal fibroadipose tissue and tracheal invasion. The surgical margin was positive for tumor. The tumor was initially interpreted as a 'poorly differentiated carcinoma, favor papillary thyroid carcinoma' and the patient was treated with post-operative radioactive iodine (Table 1). However, given the unusual morphology, the pathology slides were sent to a head and neck pathology consultant who made a diagnosis of 'metastatic papillary ductal carcinoma consistent with breast origin' being in 'association with papillary carcinoma'. Subsequently, they were reviewed and worked-up by ancillary studies at our institution and the diagnosis of MASC of the thyroid was rendered (Table 2). At the last follow-up the patient was alive with no evidence of disease by imaging 2 years after diagnosis.

\section{Materials and methods}

\section{Immunohistochemistry}

Immunohistochemistry was performed using the standard streptavidin-biotin-peroxidase procedure with the Ventana system according to the manufacturer's recommendations (Ventana medical systems, Tucson, AZ, USA). Appropriate positive and negative controls were used for each antibody. The details on the antibodies used are summarized in Table 3. 


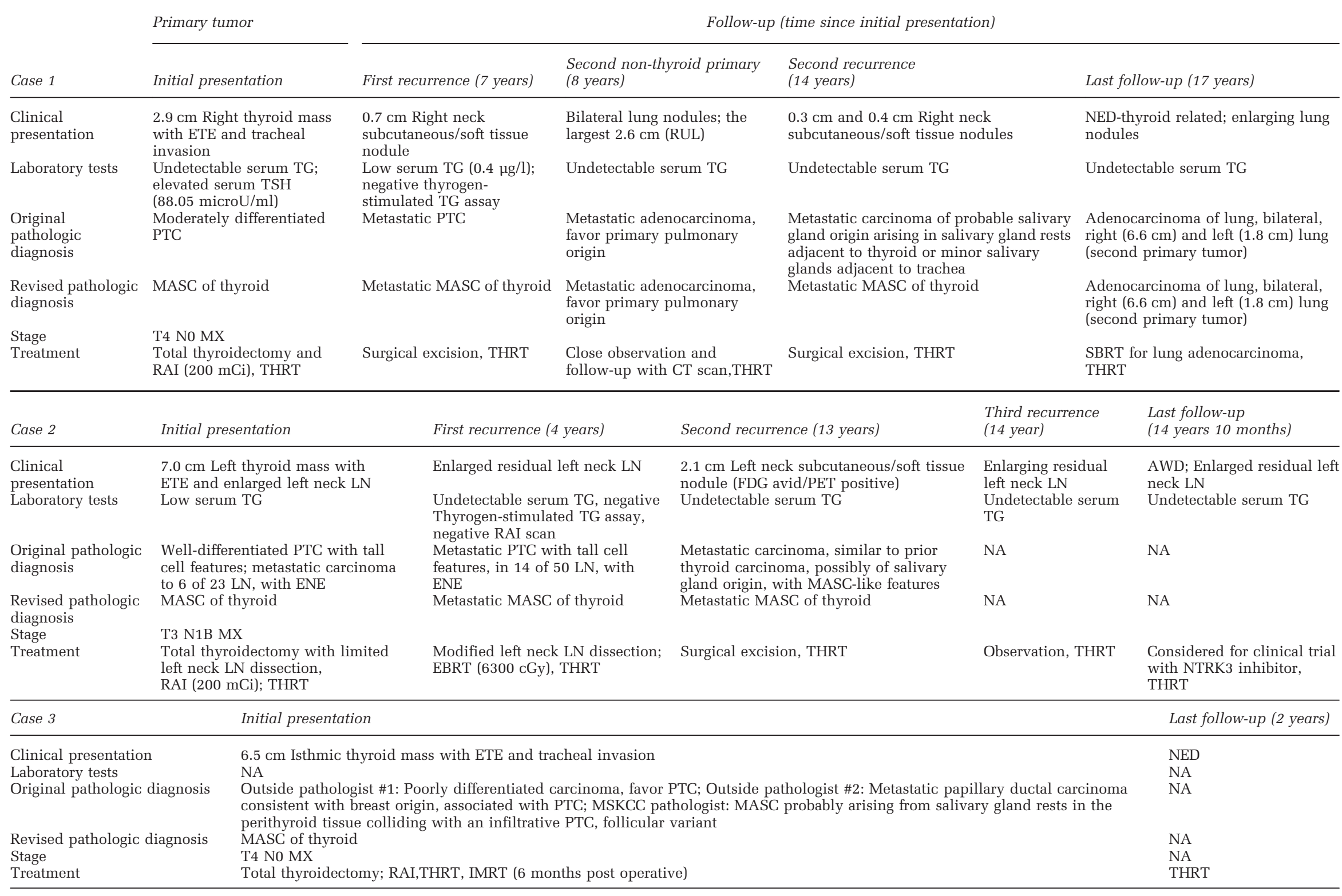

AWD, alive with disease; EBRT, external beam radiation therapy; ENE, extranodal extension; ETE, extrathyroid extension; IMRT, intensity modulated radiation therapy; LN, lymph nodes; MASC, mammary analog secretory carcinoma of salivary gland; NED, no evidence of disease; PTC, papillary thyroid carcinoma; RAI, radioactive iodine; RUL, right upper lobe; SBRT, stereotactic body radiation therapy; TG, thyroglobulin; TSH, thyroid-stimulating hormone; THRT, thyroid hormone replacement therapy. 
Table 2 Ancillary studies

\begin{tabular}{|c|c|c|c|c|c|c|c|c|c|}
\hline \multirow{3}{*}{ Ancillary study } & \multicolumn{3}{|c|}{ Case 1} & \multicolumn{4}{|c|}{ Case 2} & \multirow{2}{*}{\multicolumn{2}{|c|}{$\begin{array}{c}\text { Case } 3 \\
\text { Primary }\end{array}$}} \\
\hline & \multirow{2}{*}{ Primary } & \multirow{2}{*}{ First recurrence } & \multirow{2}{*}{ Second recurrence } & \multicolumn{2}{|l|}{ Primary } & \multirow{2}{*}{ First recurrence } & \multirow{2}{*}{$\begin{array}{l}\text { Second } \\
\text { recurrence }\end{array}$} & & \\
\hline & & & & PTC сотр. & MASC comp. & & & РTC сотр. & MASC comp. \\
\hline S-100 & $\begin{array}{l}\text { Pos (rare } \\
\text { cells) }\end{array}$ & ND & Pos (rare cells) & Neg & $\begin{array}{l}\text { Pos (rare } \\
\text { cells) }\end{array}$ & Pos (focally) & Pos (focally) & Neg & Pos \\
\hline Mammaglobin & Pos & ND & Pos & Neg & Pos & Pos & pos & Neg & Pos \\
\hline GCDFP-15 & Pos (focally) & ND & Pos (focally) & Neg & Neg & ND & Neg & Neg & Pos (focally) \\
\hline p63 & Pos (focally) & ND & ND & Neg & Pos (focally) & ND & ND & Neg & Pos \\
\hline PAX8 & Pos (weak) & ND & Pos (rare cells) & Pos & Pos (weak) & Pos (weak) & Pos & Pos & Pos (weak) \\
\hline TTF-1 & $\mathrm{Neg}$ & Neg & Neg & Pos & Neg & Neg & Neg & Pos & Neg \\
\hline Thyroglobulin & Neg & Neg & Neg & Pos & Neg & Neg & Neg & Pos & Neg \\
\hline CK7 & Pos & Pos & Pos & Pos & Pos & Pos & Pos & Pos & Pos \\
\hline CK19 & Pos & ND & Pos & Pos (weak) & Pos & Pos & Pos & $\begin{array}{l}\text { Pos (weak/very } \\
\text { focally) }\end{array}$ & Pos \\
\hline Vimentin & Pos & ND & ND & Pos (very focally) & Neg & ND & ND & Pos & Pos \\
\hline Mucicarmine & Pos & ND & Pos & Neg & Pos & ND & Pos & Neg & Neg \\
\hline Calponin & ND & ND & Neg & ND & ND & ND & Neg & Neg & Neg \\
\hline Her-2/Neu & ND & ND & Neg & ND & ND & ND & Neg & Neg & Neg \\
\hline $\mathrm{AR}$ & ND & ND & Neg & ND & ND & ND & Neg & Neg & Neg \\
\hline Ki-67 & $1-2 \%(3 / 10)$ & ND & $7 \%$ & $<1 \%$ & $<1 \%$ & ND & $2 \%$ & $<1 \%$ & $5 \%$ \\
\hline ETV6 FISH & Pos & ND & Pos & Pos & Pos & ND & Pos & Pos & Pos \\
\hline \multirow[t]{2}{*}{ MSK-IMPACT } & ND & ND & ETV6-NTRK3 & ETV6-NTRK3 & $\begin{array}{l}\text { ETV6- } \\
\text { NTRK3 }\end{array}$ & ETV6-NTRK3 & ND & & \\
\hline & & & $\begin{array}{l}\text { ETV6 P214L } \\
\text { (c.641C }>\text { T) } \\
\text { KDM5C G496R } \\
\text { (c.1486G }>\text { C) } \\
P P P 2 R 1 A \text { R } 182 W \\
(c .544 C>\text { T) }\end{array}$ & $\begin{array}{l}A P C \text { R640W } \\
\text { (c.1918C }>\text { T) } \\
H N F 1 A \text { E619K } \\
(\text { c. } 1855 \mathrm{G}>\text { A) } \\
S Y K \text { R22Q (c.65G > A) }\end{array}$ & & & & & \\
\hline
\end{tabular}

ND, not done; PTC, papillary thyroid carcinoma; MASC, mammary analog secretory carcinoma; FISH, fluorescence in situ hybridization. 
Table 3 Antibodies used for immunohistochemical studies

\begin{tabular}{llll}
\hline Antibody specificity & Clone & Dilution & Source \\
\hline S-100 & Polyclonal & 1:8000 & Dako \\
Mammaglobin & 31A5 & RTU & Ventana \\
GCDFP-15 (BR2) & D6 & $1: 1000$ & Covance \\
p63 & VS 38C & $1: 200$ & Dako \\
PAX8 & Polyclonal & $1: 100$ & Proteintech \\
TTF-1 & 8G7G3/1 & RTU & Ventana \\
Thyroglobulin & 2H11+6E1 & RTU & Cell Marque \\
CK7 & OV-TL-12/30 & $1: 1600$ & Dako \\
CK19 & RCK-108 & $1: 200$ & Dako \\
Vimentin & V9 & RTU & Ventana \\
Calponin & EP798Y & RTU & Cell Marque \\
Her-2/Neu (HER2) & 4B5 & RTU & Ventana \\
AR & AR441 & $1: 200$ & Dako \\
Ki-67 (MIB-1) & MIB-1 & $1: 200$ & Dako \\
\hline
\end{tabular}

RTU, ready to use.

\section{Fluorescence In Situ Hybridization}

Formalin-fixed paraffin-embedded tissue samples were tested for ETV6 rearrangements using a breakapart fluorescence in situ hybridization assay with a commercial ETV6 break-apart FISH probe (Abbott Molecular, USA). Four-micrometer tissue sections generated from formalin-fixed paraffin-embedded blocks of tumor specimens were pretreated by deparaffinizing in xylene and dehydrating in ethanol. Dual-color fluorescence in situ hybridization assay was conducted according to the protocol for formalin-fixed paraffin-embedded sections from Vysis/Abbott Molecular. Fluorescence in situ hybridization analysis and signal capture were conducted on a fluorescence microscope microscope (Zeiss, Jena, Germany) coupled with ISIS fluorescence in situ hybridization Imaging System (Metasystems, Heidelberg, Germany). We analyzed 100 interphase nuclei from each tumor specimen.

\section{DNA Extraction and Exon Capture Next-Generation Sequencing (MSK-IMPACT ${ }^{\mathrm{TM}}$ )}

Tumors were profiled for genomic alterations in 410 key cancer-associated genes using our deep sequencing MSK-IMPACT ${ }^{\mathrm{TM}}$ (Memorial Sloan KetteringIntegrated Mutation Profiling of Actionable Cancer Targets) assay. Custom DNA probes were designed for targeted sequencing of all exons and selected introns of 410 oncogenes, tumor suppressor genes, and members of pathways deemed actionable by targeted therapies. Genomic DNA from tumor- and patient-matched normal samples was extracted on a Hamilton Chemagic workstation using formalinfixed paraffin-embedded tissue DNA and DNA Blood kits (Perkin Elmer, Waltham, MA, USA), respectively. Genomic DNA was sheared using the Covaris E200 instrument (Covaris, Woburn, MA, USA) then subjected to sequence library preparation (Kapa Biosystems) and exon capture (NimbleGen). Barcoded sequence libraries were pooled at equi- molar concentrations and input into a single exon capture reaction, as previously described. ${ }^{11,12}$ Pooled libraries containing captured DNA fragments were subsequently sequenced on the Illumina HiSeq 2500 system as $2 \times 100 \mathrm{bp}$ paired-end reads. Samples were subjected to a series of computational quality control steps to ensure genomic concordance between tumor and normal samples from the same patient, detect the presence of tumor DNA in the normal sample, and monitor contamination involving DNA from different patients. Paired sample variant calling was performed on tumor samples and their respective matched normals to identify point mutations/single-nucleotide variants and small insertions/deletions. Annotated single-nucleotide variants and insertions/deletions calls were subjected to a series of filtering steps to ensure only high-confidence calls were admitted to the final step of manual review. ${ }^{11,13}$ Copy-number aberrations were identified by comparing sequence coverage of targeted regions in a tumor sample relative to a standard diploid normal sample.11 DELLY version 0.6.1 was used for the identification of somatic structural variants from tumor and matched normal read-pair data, as previously described. ${ }^{11,14}$

\section{Results}

\section{Microscopic Features and Immunohistochemistry}

All three tumors were large, infiltrative, and had extrathyroidal extension. They were comprised of lobules, variably-sized nests, cords, and tubules separated by dense collagenous stroma. Within the lobules and nests, tumor cells formed microcystic areas or were multilayered forming bridges, cribriform structures, true papillae as well as pseudopapillae/micropapillae. The latter lacked distinct fibrovascular cores and formed cleft-like spaces. Cytological features greatly overlapped with those usually seen in well-differentiated papillary thyroid carcinoma. However, in comparison to well-differentiated papillary thyroid carcinoma, adenocarcinoma/ MASC cells were mostly evenly spaced, showed denser eosinophilic or vacuolated cytoplasm, mainly smooth nuclear membranes, open to finely coarse chromatin, and conspicuous to prominent nucleoli (Figure 1). Nuclear features of papillary thyroid carcinoma such as nuclear membrane irregularities and grooves were subtle and focally present in each case, and rare nuclear pseudoinclusions were identified only in Case 2 and in both tumor components. High-grade tumor features such as an increased mitotic count or tumor necrosis were not identified. The proliferation index based on Ki-67 immunohistochemistry ranged from $<1$ to $7 \%$. In cases containing a minor well-differentiated papillary thyroid carcinoma component, scant colloid was present; but for the most part, secretions were scanty, dense and eosinophilic and admixed with cellular 

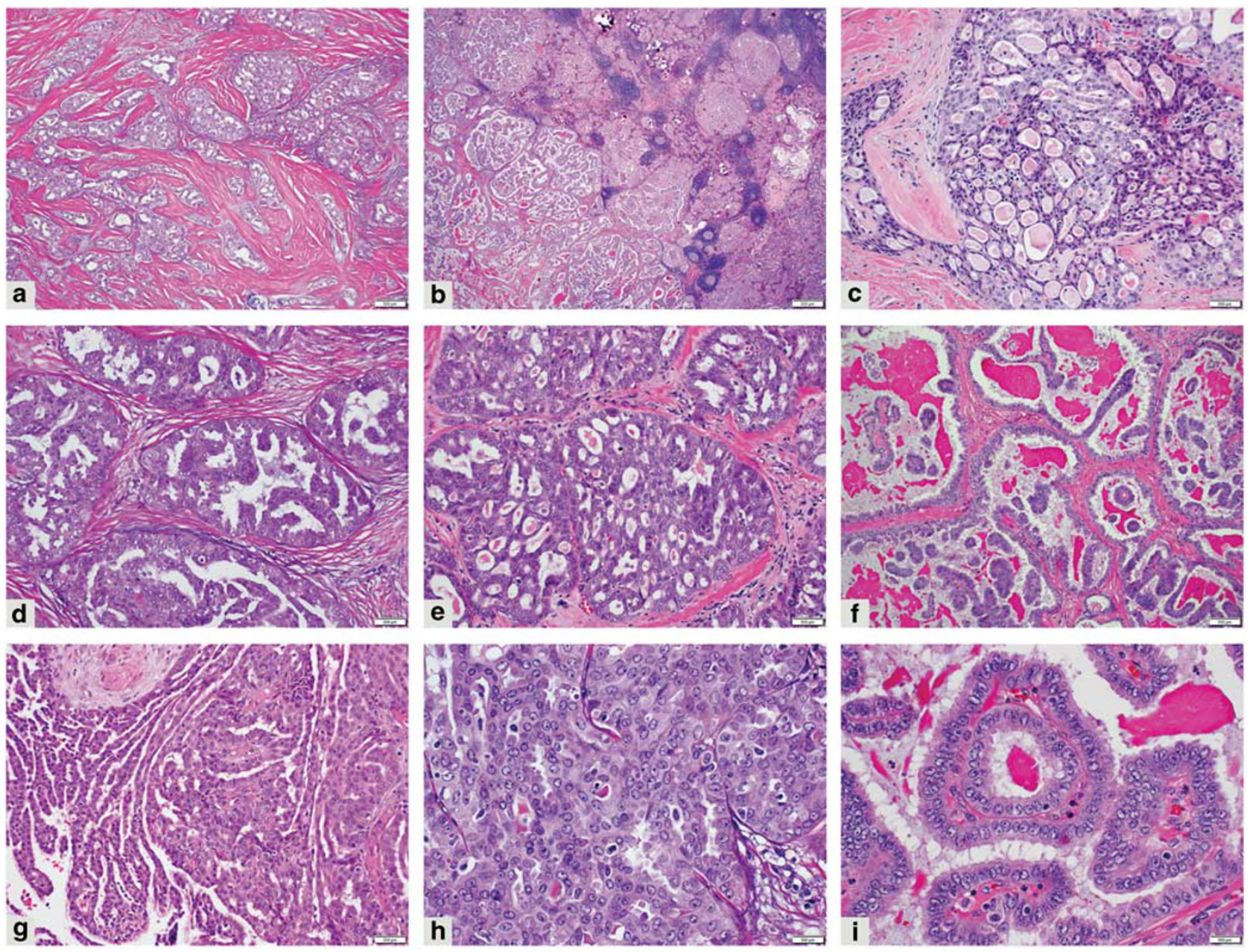

Figure 1 Morphology of mammary analog secretory carcinoma of the thyroid. Lobules, nests, and cords are divided by dense collagenous stroma (a) (100 × magnification); chronic lymphocytic thyroiditis in the background (b) (20× magnification); microcystic areas (c); multilayered epithelium forming bridges (d); cribriform structures (e) ((c-e), $200 \times$ magnification); true papillae in cystic spaces and with associated dense eosinophilic secretions (f) (100X magnification); pseudopapillae/micropapillae with cleft-like spaces (g) (200× magnification); tumor cells with eosinophilic cytoplasm, enlarged nuclei with little overlapping, clear to finely clumped/coarse chromatin and prominent nucleoli (h,i) $(400 \times$ magnification).

debris. The adjacent benign thyroid parenchyma showed chronic lymphocytic thyroiditis in each of the three cases. In Cases 2 and 3 there was a welldifferentiated papillary thyroid carcinoma component closely associated with the adenocarcinoma/ MASC part of the tumor (Figures 2 and 3). In Case 2, it was a classic well-differentiated papillary thyroid carcinoma component. However, no well-differentiated papillary thyroid carcinoma part was identified in either of the subsequent metastases in Case 2. In Case 3 the well-differentiated papillary thyroid carcinoma was the follicular variant. In both instances, the immunohistochemical work-up confirmed the morphological impression, showing distinct immunoprofiles of each respective tumor component (Table 2). No well-differentiated papillary thyroid carcinoma component was found in Case 1. All adenocarcinoma/MASC components were positive for mammaglobin, GCDFP-15, S-100 protein, p63 (focally), and weakly positive for PAX8, whereas negative for TTF-1 and thyroglobulin. The associated well-differentiated papillary thyroid carcinoma components showed essentially the inverse staining pattern (eg, TTF-1 and thyroglobulin positivity) with the exception of PAX8 that was positive in both tumor components but was notably stronger in well-differentiated papillary thyroid carcinoma than in adenocarcinoma/MASC (Table 2).

\section{Cytogenetics}

Fluorescence in situ hybridization was performed on all three cases, revealing ETV6 rearrangement in 65, 83 and $83 \%$ tumor cells, respectively (Table 2). Importantly, in Cases 2 and 3, the ETV6 rearrangement was detected in both adenocarcinoma/MASC and welldifferentiated papillary thyroid carcinoma component, further supporting them being components of the same tumor and having common cell of origin (Figure 2). 

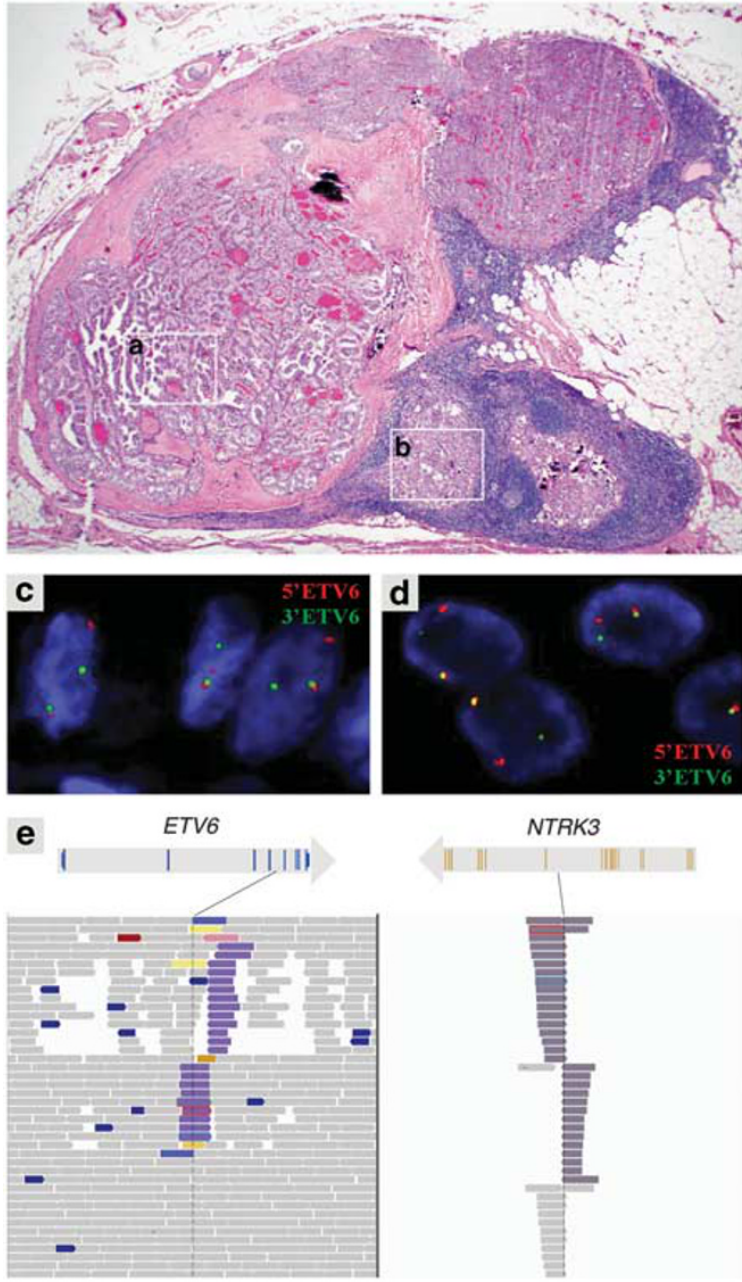

NTRK3
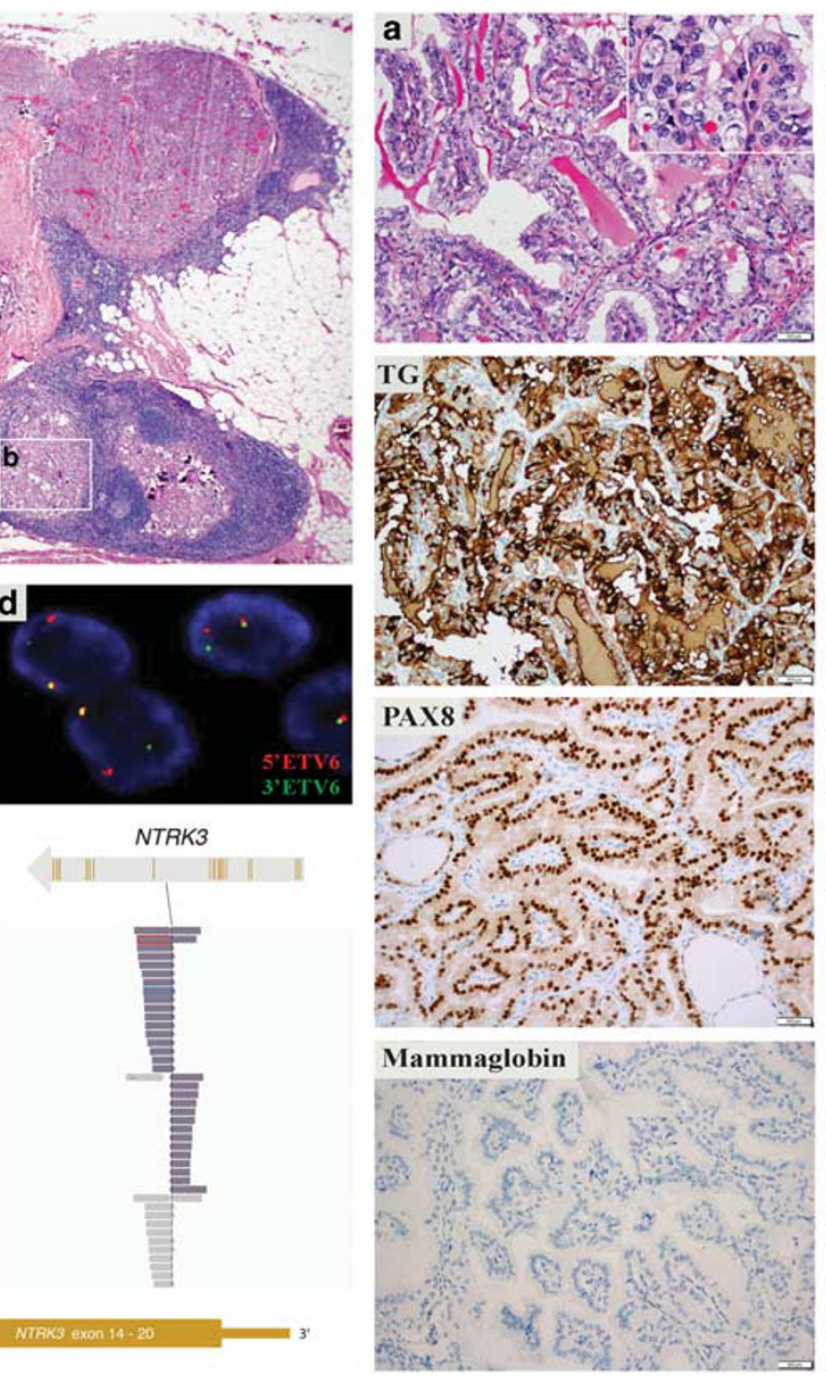
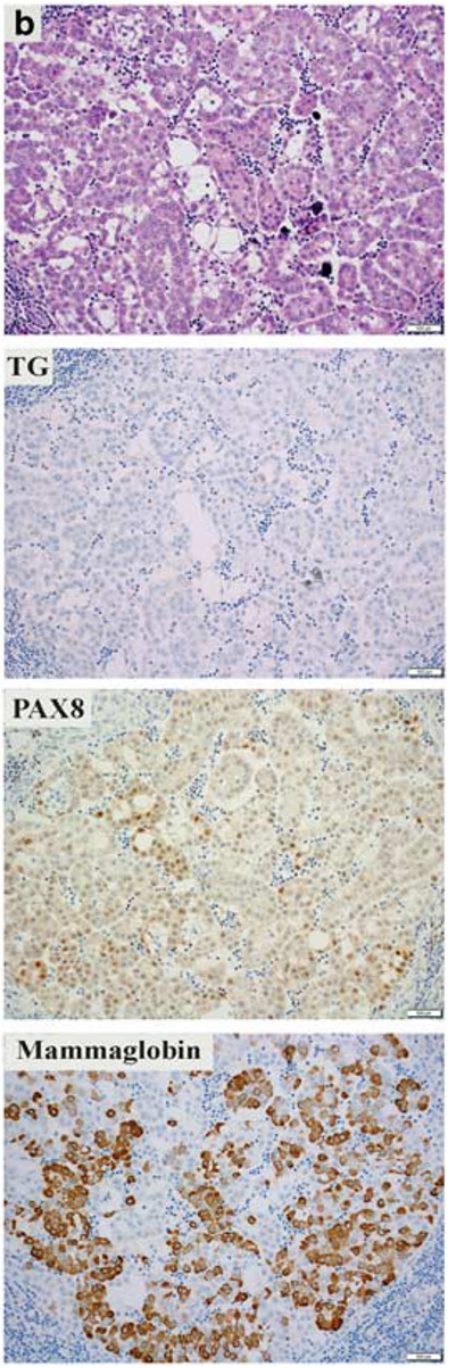

Figure 2 Case 2. A perithyroidal lymph node metastasis with well-differentiated papillary thyroid carcinoma (a) and mammary analog secretory carcinoma (b) tumor components (left upper; $40 \times$ magnification). Right side panel: well-differentiated papillary thyroid carcinoma component (column A, $200 \times$ magnification; with papillary thyroid carcinoma papillary thyroid carcinoma nuclear features, H\&E inset, $400 \times$ magnification) stained positive for thyroglobulin (TG), PAX8, and negative for mammaglobin, and mammary analog secretory carcinoma component (column B, $200 \times$ magnification) stained negative for TG, weakly positive for PAX8, and positive for mammaglobin. Both tumor components show ETV6 rearrangement by fluorescence in situ hybridization (well-differentiated papillary thyroid carcinoma) (c) and, mammary analog secretory carcinoma (d). MSK-IMPACT assay: exons 1-4 of ETV6 gene are fused with the exons 14-20 of NTRK3 gene resulting in ETV6-NTRK3 fusion transcript (e).

\section{Molecular Profile}

The last recurrence of Case 1 and primary tumor and two recurrences of Case 2 were subjected to MSKIMPACT. In each tested sample, a balanced rearrangement $\mathrm{t}(12 ; 15)(\mathrm{p} 13.2 ; \mathrm{q} 25.3)$ was identified resulting in an ETV6-NTRK3 fusion transcript where exons 14 of ETV6 were fused with exons 14-20 of NTRK3 (Figure 2). Additional mutations involving ETV6, $K D M 5 C$, and PPP2R1A genes were identified in Case 1 at 32,28 , and $2 \%$ variant frequencies, respectively (Table 2). In the primary tumor of Case 2 in addition to the oncogenic ETV6-NTRK3 fusion three other somatic single-nucleotide variants were detected at low variant frequencies (5\%; Table 2).

\section{Discussion}

MASC of the salivary glands, recently described and distinguished from acinic cell carcinoma, is histologically characterized by uniform cells with vesicular nuclei and eosinophilic vacuolated cytoplasm, arranged in tubular, microcystic, and solid growth patterns often divided by dense fibrous septa, and with abundant PAS-positive colloid-like secretory material. MASC is typically positive for S-100 protein, mammaglobin, vimentin, and cytokeratins, namely CK7 and CK19, and can be focally positive for p63. Similar to its breast counterpart, MASC harbors a recurrent balanced chromosomal translocation $\mathrm{t}(12 ; 15)(\mathrm{p} 13 ; \mathrm{q} 25)$ leading to ETV6-NTRK3 

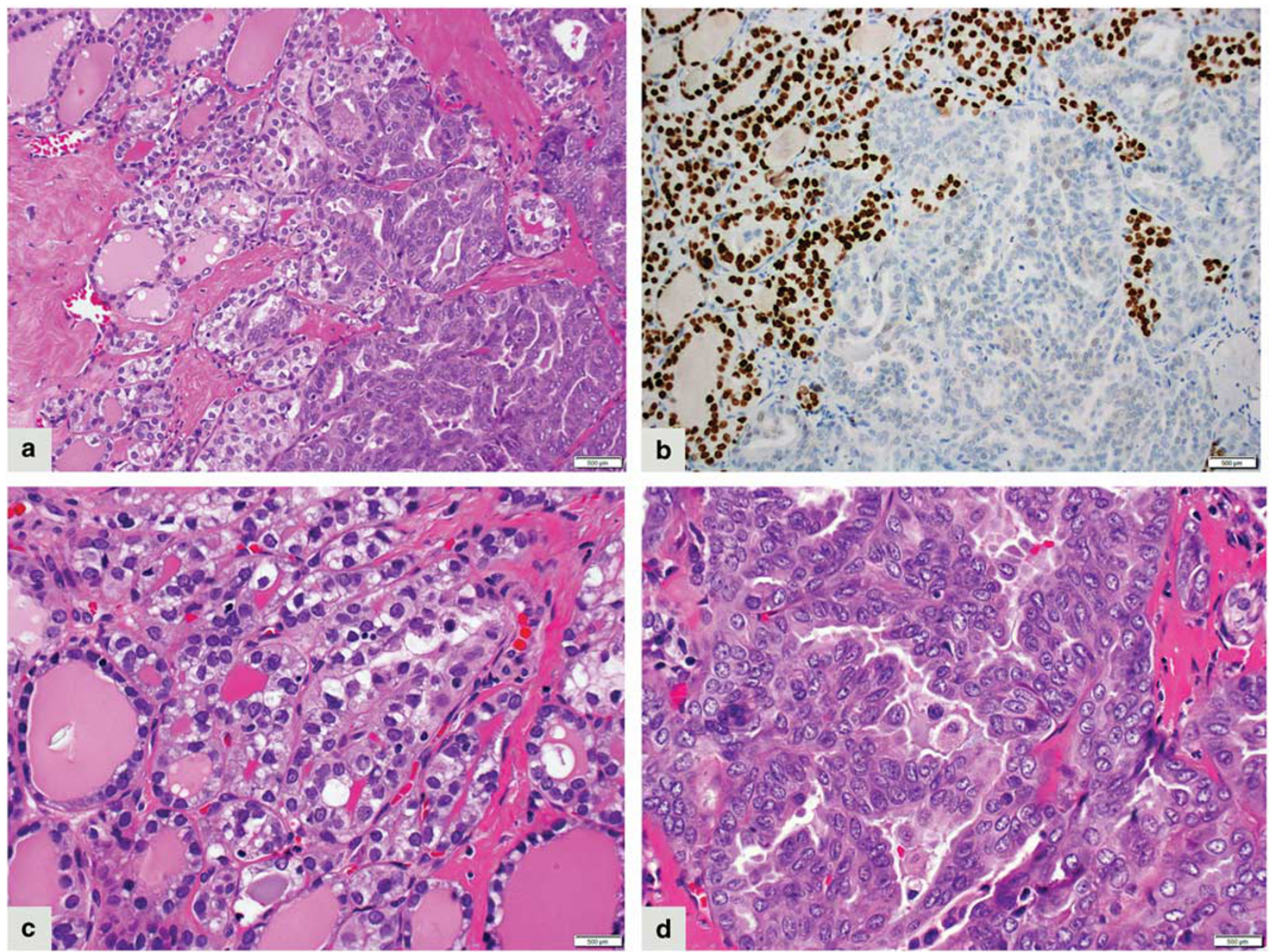

Figure 3 Case 3. A smooth transition between well-differentiated papillary thyroid carcinoma, follicular variant (left side) and mammary analog secretory carcinoma component (right side) (a) (200x magnification); TTF-1 immunostain is positive in well-differentiated papillary thyroid carcinoma (left side) and negative in mammary analog secretory carcinoma component (right side) (b) (200 $\times$ magnification). Papillary thyroid carcinoma is composed of colloid-filled follicles and tumor cells show pale eosinophilic cytoplasm, enlarged nuclei with irregular nuclear membranes, open chromatin and absent to inconspicuous nucleoli (c) $(400 \times$ magnification). The transition to the mammary analog secretory carcinoma phenotype is characterized by lack of colloid, denser eosinophilic cytoplasm, enlarged nuclei with open chromatin, and conspicuous to prominent nucleoli (d) $(400 \times$ magnification).

gene fusion. ${ }^{1,15}$ Most of the reported cases showed the behavior of a low-grade adenocarcinoma, with about $15 \%$ recurrence rate and $15 \%$ rate of lymph node metastases. ${ }^{16}$

Here, we report three cases of primary adenocarcinomas of thyroid gland occurring in two women and one man, age 47-72 years (average 61 years). There was no history of radiation exposure in any patient. All three patients presented with a high $\mathrm{T}$ stage, infiltrative, locally aggressive tumors with extrathyroidal extension, arising in a background of chronic lymphocytic thyroiditis. In two cases, a well-differentiated papillary thyroid carcinoma was present as a minor tumor component, whereas one case was entirely comprised of adenocarcinoma. Histologically, they appeared to be of low-grade, resembling MASC of salivary gland and its breast counterpart. 1,17,18 Although immunoreactivity for S-100 protein and mammaglobin were compatible with a salivary gland/breast-like phenotype, positive labeling for PAX8, a marker of thyroid lineage, supported these tumors to represent primary thyroid carcinomas and not metastases from a primary salivary gland or breast carcinoma. ${ }^{19-22}$ Importantly, similar to MASC of salivary gland, the ETV6 gene rearrangement was detected by fluorescence in situ hybridization in all three tumors, and the presence of the ETV6-NTRK3 fusion product was subsequently confirmed by next-generation sequencing assay in two tested cases. Therefore, based on the above findings and the lack of salivary gland and breast involvement in these patients, we propose these carcinomas to represent primary MASCs of the thyroid gland, a previously unrecognized entity. Recently, in their series of MASC of salivary gland Stevens et al. ${ }^{23}$ reported a case of 55-year-old woman with MASC involving the thyroid and extending into the adjacent perilaryngeal soft tissues. The authors 
suggested ectopic salivary glands as a primary site of origin. Although this still remains a possibility, based on our current experience we believe that this previously described case would likely represent another example of primary MASC of the thyroid gland.

ETV6-NTRK3 fusion was recently reported to be present in about $14 \%$ of radiation-induced welldifferentiated papillary thyroid carcinoma, and in up to $2 \%$ sporadic well-differentiated papillary thyroid carcinoma. Among sporadic cases, classical type and follicular variants were described.6,7 Two of our cases showed a minor well-differentiated papillary thyroid carcinoma component. In Case 2, welldifferentiated papillary thyroid carcinoma was of the classical type, and in Case 3 it was follicular variant papillary thyroid carcinoma (Figure 3). Detection of ETV6 rearrangement in both, the MASC-like and well-differentiated papillary thyroid carcinoma tumor elements is supportive of both components belonging to the same neoplastic process and having a common cell of origin. Given the histological evidence of a smooth transition from one phenotype to another ie, from well-differentiated papillary thyroid carcinoma to MASC-like tumor (best seen in Case 3, Figure 3) and the finding of PAX8 expression in both components, these thyroid MASCs likely represent a thyroid carcinoma of follicular cell origin, ie, a well-differentiated papillary thyroid carcinoma that underwent divergent differentiation into another tumor type. In Case 1 no well-differentiated papillary thyroid carcinoma component was identified and the serum thyroglobulin was undetectable at first presentation consistent with the absence of the thyroglobulinproducing tumor component. Therefore, based on our findings and in view of the prior reports of ETV6 rearranged well-differentiated papillary thyroid carcinoma, we suggests that primary ETV6NTRK3-driven thyroid carcinomas may not just represent a molecular subtype of well-differentiated papillary thyroid carcinoma but would also include a salivary gland-like adenocarcinoma with or without associated well-differentiated papillary thyroid carcinoma component. We propose that these carcinomas should be primarily viewed as MASC of the thyroid gland for the following reasons: (1) morphologically, the well-differentiated papillary thyroid carcinoma component was minor in Cases 2 and 3, and was completely absent in Case 1; (2) clinically, these carcinomas did not behave as well-differentiated papillary thyroid carcinoma but rather as low-grade salivary gland-type adenocarcinomas; (3) thyroglobulin levels were undetectable at the time of histologically confirmed recurrence in Cases 1 and 2; and (4) thyrogen-stimulated assays were negative in both Case 1 and 2 .

Despite the multiple recurrences in both Case 1 and 2, both patients were alive after 17 and 14 years, respectively, and the third patient was alive and free of disease after 2 years. These clinical features suggest a relatively indolent clinical behavior even in the absence of an effective systemic treatment. However, the presence of an ETV6-NTRK3 fusion protein as a molecular target could expand the treatment options for patients with systemic disease. Recently we reported a case of metastatic MASC of the salivary gland with a positive response to the pan-Trk inhibitor entrectinib. ${ }^{24}$ These findings could provide a rationale for taking a similar approach in patients with the MASC of the thyroid presenting with metastatic or recurrent disease.

Distinguishing MASC from well-differentiated papillary thyroid carcinoma can be very challenging, particularly from the one harboring the ETV6-NTRK3 fusion. Given the marked morphologic and genetic similarity between well-differentiated papillary thyroid carcinoma and MASC we believe that the diagnosis of MASC needs to be strongly considered if the ETV6 rearrangement is detected in a thyroid tumor with papillary morphology and especially in patients without history of radiation exposure. Aside from well-differentiated papillary thyroid carcinoma, the differential diagnosis may include poorly differentiated thyroid carcinoma, metastatic breast carcinoma, or metastatic MASC of the salivary gland. Poorly differentiated thyroid carcinoma requires detection of at least 5 mitoses per 10 highpower fields and/or tumor necrosis in the setting of TTF-1 and thyroglobulin positivity. ${ }^{25}$ However, although our cases exhibited histologic features of a low-grade carcinoma, we cannot completely exclude the possibility that there are high-grade primary MASCs of the thyroid, similar to such cases seen in the salivary glands. ${ }^{24,26}$ Therefore increased mitotic activity or tumor necrosis by themselves may not necessarily exclude MASC in thyroid. Despite the marked morphological overlap between welldifferentiated papillary thyroid carcinoma and primary MASC of thyroid, especially at cytologic level, features such as multilayered tufting tumor epithelium with bridges, cribriforming, microcystic areas, psuedopapillae/micropapillae and relatively evenly spaced nuclei with conspicuous to prominent nucleoli may raise a possibility of MASC arising in the thyroid gland. This should prompt an initial immunohistochemical work-up with thyroglobulin and mammaglobin, and if appropriate, an addition of immunostains for TTF-1, PAX8, and S-100 protein. A positive PAX8 immunostain and no clinical history of breast or salivary gland primary tumor may be sufficient to rule out salivary gland and breast carcinoma metastasis. Finally, detection of ETV6NTRK3 fusion transcript would not only support the diagnosis but may become essential if further management with Trk inhibitors is considered. Our study shows that MASC of the thyroid gland is not a thyroglobulin-producing tumor, and in the setting of metastatic disease these patients are unlikely to respond to radioactive iodine therapy.

In addition to the oncogenic ETV6-NTRK3 fusion, targeted next-generation sequencing of the two cases 
revealed a few additional genetic events in each case suggesting a relatively 'quiet' tumor genome. A comprehensive sequential mutation profiling of the primary tumor and the subsequent recurrences may help elucidate the genetic background associated with intratumoral heterogeneity and tumor progression. All three single-nucleotide variants $A P C$ R640W, HNF1A E619K, and SYK R22Q co-occurring with the ETV6-NTRK3 in Case 2 were present at low variant frequencies in the primary tumor sample and were absent in subsequent recurrences where the ETV6-NTRK3 fusion was the only detected genetic alteration. These findings suggest that these additional genetic alterations were likely subclonal events present only in a minor subpopulation of the tumor cells. In addition, their absence in the subsequent metastases suggests these singlenucleotide variants unlikely to be functionally significant or associated with the tumor recurrence and progression. This further emphasizes the oncogenic role of the ETV6-NTRK3 fusion gene in this tumor type. Similarly, in Case 1 detection of PPP2R1A R182W at only $2 \%$ variant frequency also favors a subclonal mutation. Interestingly, in Case 1 in addition to the rearrangement ETV6 gene was also affected by a somatic P214L mutation. Although the ETV6 P214L and KDM5C G496R were both present at relatively high variant frequencies consistent with their presence in the majority of tumor cells, their potential functional significance remains uncertain at this point.

\section{Conclusion}

Here, we report three cases of primary MASC of the thyroid gland occurring in middle-aged patients. All three cases were found to harbor ETV6 rearrangement and two tested cases were confirmed to harbor the ETV6-NTRK3 gene fusion. In addition to the ETV6-NTRK3 these tumors were characterized by a few additional somatic genetic alterations suggesting a relatively 'quiet' tumor genome. Histologically, MASC of thyroid appears to be a low-grade tumor that can be associated with a well-differentiated papillary thyroid carcinoma component. Clinically, these tumors showed frequent recurrences but longterm survival. Pathologists should be aware of MASC in thyroid, and where the morphological features suggest so, ancillary studies including molecular testing for ETV6-NTRK3 rearrangement should be performed. In the appropriate clinical setting, patients with MASC of the thyroid gland may potentially benefit from pan-Trk Trk molecular-targeted therapy.

\section{Acknowledgments}

We thank Allyne Manzo, our expert photographer from the Department of Pathology, MSKCC for her assistance in arranging the microphotographs. Research reported in this publication was supported in part by the Cancer Center Support Grant of the National Institutes of Health/National Cancer Institute under award number P30CA008748. The content is solely the responsibility of the authors and does not necessarily represent the official views of the National Institutes of Health.

\section{Disclosure/conflict of interest}

The authors declare no conflict of interest.

\section{References}

1 Skálová A, Vanecek T, Sima R, et al. Mammary analogue secretory carcinoma of salivary glands, containing the ETV6-NTRK3 fusion gene: a hitherto undescribed salivary gland tumor entity. Am J Surg Pathol 2010;34:599-608.

2 Tognon C, Knezevich SR, Huntsman D, et al. Expression of the ETV6-NTRK3 gene fusion as a primary event in human secretory breast carcinoma. Cancer Cell 2002;2:367-376.

3 Knezevich SR, McFadden DE, Tao W, et al. A novel ETV6-NTRK3 gene fusion in congenital fibrosarcoma. Nat Genet 1998;18:184-187.

4 Rubin BP, Chen CJ, Morgan TW, et al. Congenital mesoblastic nephroma $\mathrm{t}(12 ; 15)$ is associated with ETV6-NTRK3 gene fusion: cytogenetic and molecular relationship to congenital (infantile) fibrosarcoma. Am J Pathol 1998;153:1451-1458.

5 Eguchi M, Eguchi-Ishimae M, Tojo A, et al. Fusion of ETV6 to neurotrophin-3 receptor TRKC in acute myeloid leukemia with $\mathrm{t}(12 ; 15)(\mathrm{p} 13 ; \mathrm{q} 25)$. Blood 1999;93:1355-1363.

6 Leeman-Neill RJ, Kelly LM, Liu P, et al. ETV6-NTRK3 is a common chromosomal rearrangement in radiationassociated thyroid cancer. Cancer 2014;120:799-807.

7 Cancer Genome Atlas Research N. Integrated genomic characterization of papillary thyroid carcinoma. Cell 2014;159:676-690.

8 Rhatigan RM, Roque JL, Bucher RL. Mucoepidermoid carcinoma of the thyroid gland. Cancer 1977;39: 210-214.

9 Chan JK, Albores-Saavedra J, Battifora H, et al. Sclerosing mucoepidermoid thyroid carcinoma with eosinophilia. A distinctive low-grade malignancy arising from the metaplastic follicles of Hashimoto's thyroiditis. Am J Surg Pathol 1991;15:438-448.

10 Sim SJ, Ro JY, Ordonez NG, et al. Sclerosing mucoepidermoid carcinoma with eosinophilia of the thyroid: report of two patients, one with distant metastasis, and review of the literature. Hum Pathol 1997;28: 1091-1096.

11 Cheng DT, Mitchell TN, Zehir A, et al. MSK-IMPACT: a hybridization capture-based next generation sequencing clinical assay for solid tumor molecular oncology. J Mol Diagn 2015;17:251-264.

12 Won HH, Scott SN, Brannon AR, et al. Detecting somatic genetic alterations in tumor specimens by exon capture and massively parallel sequencing. J Vis Exp 2013;80:e50710. 
13 Robinson JT, Thorvaldsdóttir H, Winckler W, et al. Integrative genomics viewer. Nat Biotechnol 2011;29:24-26.

14 Rausch T, Zichner T, Schlattl A, et al. DELLY: structural variant discovery by integrated paired-end and split-read analysis. Bioinformatics 2012;28:i333-i339.

15 Skálová A. Mammary analogue secretory carcinoma of salivary gland origin: an update and expanded morphologic and immunohistochemical spectrum of recently described entity. Head Neck Pathol 2013;7(Suppl 1): 30-36.

16 Balanzá R, Arrangoiz R, Cordera F, et al. Mammary analog secretory carcinoma of the parotid gland: a case report and literature review. Int J Surg Case Rep 2015;16:187-191.

17 Chiosea SI, Griffith C, Assaad S, et al. Clinicopathological characterization of mammary analogue secretory carcinoma of salivary glands. Histopathology 2012;61:387-394.

18 Bishop J, Yonescu R, Bastista D, et al. Most non-parotid actinic cell carcinomas represent mammary analog secretory carcinomas. Am J Surg Pathol 2013;37: 1053-1057.

19 Bhargava R, Beriwal S, Dabbs DJ. Mammaglobin vs GCDFP-15: an immunohistologic validation survey for sensitivity and specificity. Am J Clin Pathol 2007;127: 103-113.

20 Fabbro D, Di Loreto C, Beltrami CA, et al. Expression of thyroid-specific transcription factors TTF-1 and
PAX-8 in human thyroid neoplasms. Cancer Res 1994; 54:4744-4749.

21 Ros P, Rossi DL, Acebrón A, et al. Thyroid-specific gene expression in the multi-step process of thyroid carcinogenesis. Biochimie 1999;81:389-396.

22 Nonaka D, Tang Y, Chiriboga L, et al. Diagnostic utility of thyroid transcription factors Pax8 and TTF-2 (FoxE1) in thyroid epithelial neoplasms. Mod Pathol 2008;21: 192-200.

23 Stevens TM, Kovalovsky AO, Velosa C, et al. Mammary analog secretory carcinoma, low-grade salivary duct carcinoma, and mimickers: a comparative study. Mod Pathol 2015;28:1084-1100.

24 Drilon A, Li G, Dogan S, et al. What hides behind the MASC: clinical response and acquired resistance to entrectinib after ETV6-NTRK3 identification in a mammary analogue secretory carcinoma (MASC). Ann Oncol 2016;27:920-926.

25 Hiltzik D, Carlson DL, Tuttle RM, et al. Poorly differentiated thyroid carcinomas defined on the basis of mitosis and necrosis: a clinicopathologic study of 58 patients. Cancer 2006;106:1286-1295.

26 Skálová A, Vanecek T, Majewska H, et al. Mammaryanalogue secretory carcinoma of salivary glands with high-grade transformation: report of 3 cases with the ETV6-NTRK3 gene fusion and analysis of TP53, betacatenin, EGFR, and CCND1 genes. Am J Surg Pathol 2014;38:23-33. 